\title{
Surface differential rotation of IL Hya from time-series Doppler images
}

\author{
Zsolt Kövári ${ }^{1}$, Levente Kriskovics ${ }^{1}$, Katalin Oláh ${ }^{1}$ Krisztián Vida $^{1}$ \\ János Bartus ${ }^{1}$, Klaus G. Strassmeier ${ }^{2}$ and Michael Weber ${ }^{2}$ \\ Konkoly Observatory, \\ ${ }^{1}$ Konkoly Thege út 15-17., H-1121, Budapest, Hungary \\ email: kovari, kriskovics, olah, vida, bartus@konkoly.hu \\ ${ }^{2}$ Leibniz Institute for Astrophysics Potsdam, \\ An der Sternwarte 16, 14482 Potsdam, Germany \\ email: kstrassmeier, mweber@aip.de
}

\begin{abstract}
We present a time-series Doppler imaging study of the K-subgiant component in the RS CVn-type binary system IL Hya $\left(P_{\text {orb }}=12.905 \mathrm{~d}\right)$. From re-processing the unique longterm spectroscopic dataset of 70 days taken in 1996/97, we perform a thorough cross-correlation analysis to derive surface differential rotation. As a result we get solar-type differential rotation with a shear value $\alpha$ of 0.05 , in agreement with preliminary suggestions from previous attempts. A possible surface pattern of meridional circulation is also detected.
\end{abstract}

Keywords. stars: activity, stars: imaging, stars: individual (IL Hya), stars: spots, stars: late-type

\section{Time-series Doppler images of IL Hya}

IL Hya is a double-lined binary star (K0IV + G8V), a typical RS CVn-type system orbiting with a period of 12.905 days. Our time-series spectroscopic dataset were obtained during a 70-night long observing run at NSO in 1996/97. From that we reconstruct 30 time-series Doppler images for two favoured mapping lines (Fe I-6430 and Ca I-6439) using our image reconstruction code TEMPMAP (Rice et al. 1989). Adopted astrophysical parameters are listed in Table 1. As samples from the reconstructions, combined $(\mathrm{Fe}+\mathrm{Ca})$ maps are shown in Fig. 1, indicating significant changes of the spotted surface over a few rotation cycles.

\section{Surface differential rotation and meridional flow}

To measure surface DR we employ our method called 'ACCORD' (acronym from Average Cross-CORrelation of consecutive Doppler images), based on averaging crosscorrelation function (ccf) maps of subsequent Doppler images. This way the surface differential rotation (hereafter DR) pattern in the ccf-maps could be enhanced, while
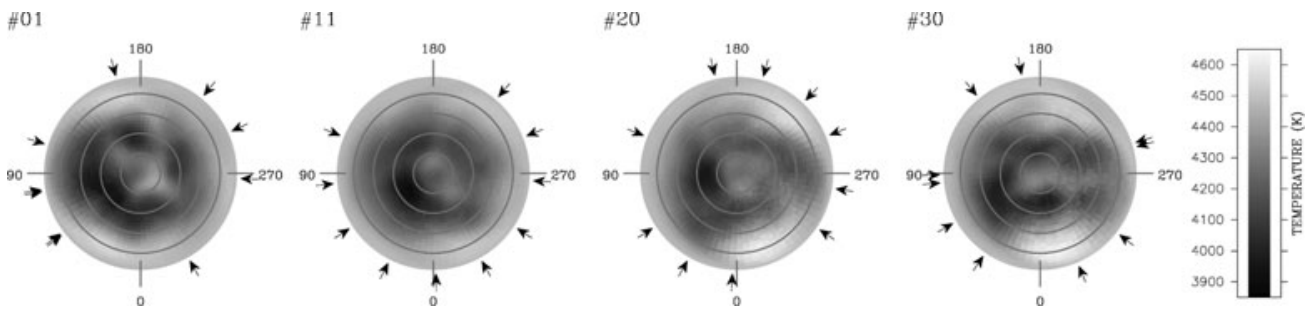

Figure 1. Time evolution of the spotted surface of IL Hya from time-series Doppler imaging. 
Table 1. Astrophysical chart of IL Hya based on Weber \& Strassmeier (1998)

\begin{tabular}{ll}
\hline Spectral type & K0IV $(+\mathrm{G} 8 \mathrm{~V})$ \\
$\log g$ & $2.5 \pm 0.5$ \\
$T_{\text {eff }}[\mathrm{K}]$ & $4500 \pm 250$ \\
$B-V[\mathrm{mag}]$ & $1.012 \pm 0.010$ \\
$V-I[\mathrm{mag}]$ & $0.99 \pm 0.01$ \\
Distance ${ }^{a}[\mathrm{pc}]$ & $105.9 \pm 5.6$ \\
$v \sin i\left[\mathrm{~km} \mathrm{~s}^{-1}\right]$ & $26.5 \pm 1.0$ \\
Inclination $[\mathrm{deg}]$ & $55 \pm 5$ \\
$P_{\text {orb }[\text { days }}$ & $12.905 \pm 0.004$ \\
Radius $^{a}[\mathrm{R} \odot]$ & $8.1 \pm 0.9$ \\
Microturbulence $\left[\mathrm{km} \mathrm{s}^{-1}\right]$ & 2.0 \\
Macroturbulence $\left[\mathrm{km} \mathrm{s}^{-1}\right]$ & 4.0 \\
Chemical abundances & 0.9 dex below solar \\
Mass $\left[\mathrm{M}_{\odot}\right]$ & $\approx 2.2$ \\
\hline
\end{tabular}

${ }^{a}$ based on Hipparcos data
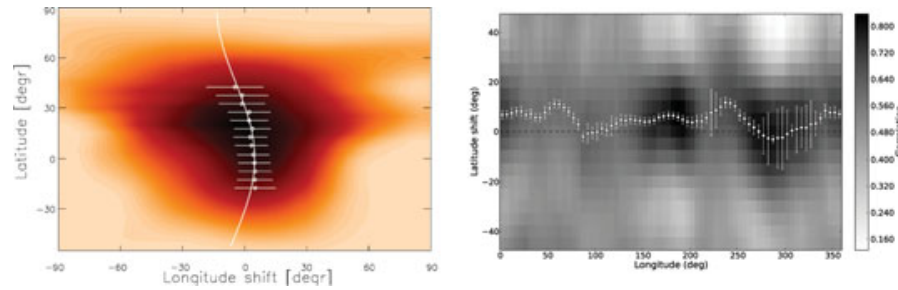

Figure 2. Averaged cross-correlations clearly reveal solar-type DR pattern (left) and common poleward drifting of spots (right).

the unwanted effect of stochastic spot changes are supressed (see Kövári et al. 2004, 2007 for details). Applying ACCORD yields solar-type rotation law in the form of $\Omega(\beta)=\Omega_{\mathrm{eq}}-\Delta \Omega \sin ^{2} \beta$ with an equatorial angular velocity $\Omega_{\mathrm{eq}}$ of $28.28 \pm 0.03 \mathrm{deg} / \mathrm{day}$ and $\Delta \Omega=\Omega_{\mathrm{eq}}-\Omega_{\mathrm{pole}}$ of $-1.43 \pm 0.15 \mathrm{deg} / \mathrm{day}$, corresponding with a surface shear $\alpha=\Delta \Omega / \Omega_{\text {eq }}$ of $0.05 \pm 0.01$ (see the fitted average ccf-map in the left panel of Fig. 1 ). This shear is consistent with the value of $\alpha=0.03 \pm 0.02$ derived by using a different method for a different dataset taken in 1988 (Kövári \& Weber 2004). Regarding the reliability of the results read the other paper by Kővári et al. in this proceedings.

Latitudinal motion of spots can also be quantified by ACCORD. For this we use only the hemisphere of the visible pole. For a detailed description of the method see Kővári et al. (2007). The resulting latitudinal correlation pattern (right panel in Fig. 2) can be converted into an average poleward surface velocity field of $0.5 \pm 0.1 \mathrm{~km} / \mathrm{s}$, that could be interpreted as the surface pattern of a single-cell meridional circulation.

\section{Acknowledgements}

This work has been supported by the Hungarian Science Research Program OTKA K-81421, the Lendület-2009 and Lendület-2012 Young Researchers' Programs of the Hungarian Academy of Sciences and by the HUMAN MB08C 81013 grant of the MAG Zrt.

\section{References}

Kővári, Zs., Strassmeier, K. G., Granzer, T., et al. 2004, A\&\&A, 417, 1047

Kővári, Zs. \& Weber, M. 2004, PADEU Vol. 14, 221

Kővári, Zs., Bartus, J., Strassmeier, K. G., et al. 2007, A\&A, 474, 165

Rice, J. B., Wehlau, W. H., \& Khokhlova, V. L. 1989, A\& A, 208, 179

Weber, M. \& Strassmeier, K. G. 1998, A\&A, 330, 1029 